\title{
Efficacy of a telephone outcall program to reduce caregiver burden among caregivers of cancer patients [PROTECT]: a randomised controlled trial
}

Leila Heckel $^{1^{*}}$ (D), Kate M. Fennell ${ }^{3,4,5}$, John Reynolds ${ }^{6}$, Anna Boltong ${ }^{7,8}$, Mari Botti ${ }^{1,9}$, Richard H. Osborne ${ }^{2}$, Cathrine Mihalopoulos ${ }^{2}$, Jacquie Chirgwin ${ }^{10,11}$, Melinda Williams ${ }^{12}$, Cadeyrn J. Gaskin ${ }^{13}$, David M. Ashley $y^{1,14}$ and Patricia M. Livingston ${ }^{1}$

\begin{abstract}
Background: Informal caregivers provide extended support to people with cancer but they receive little support from the health care system to assist them in their caring role. The aim of this single-blind, multi-centre, randomised controlled trial was to test the efficacy of a telephone outcall program to reduce caregiver burden and unmet needs, and improve psychological well-being among cancer caregivers, as well as evaluating the potential impact on patient outcomes.

Methods: Cancer patient/caregiver dyads ( $N=216)$ were randomised to a telephone outcall program $(n=108)$ or attention control group $(n=108)$. The primary outcome was self-reported caregiver burden. Secondary endpoints included depressive symptoms, unmet needs, self-esteem, self-empowerment, and health literacy. Data were collected at baseline and at both 1 and 6 months post-intervention. An intention to treat analysis was performed.

Results: The intervention had no effect on the primary outcome (caregiver burden), but reduced the number of caregiver unmet needs (intervention group baseline, mean $=2.66,95 \%$ confidence interval (Cl) [1.91-3.54]; intervention group 1 month post intervention, mean $=0.85,95 \% \mathrm{Cl}$ [0.42-1.44]; control group baseline, mean $=1.30$ 95\% Cl [0.80-1.94], control group 1 month post intervention, mean $=1.0295 \% \mathrm{Cl}$ [0.52-1.69]; $p=0.023$ ). For caregivers at risk for depression, the intervention had a significant effect on caregivers' confidence in having sufficient information to manage their health $(p=0.040)$. No effects were found for patients' depressive symptoms, unmet needs, self-empowerment, and other health literacy domains.
\end{abstract}

Conclusions: While caregiver burden was not reduced, the outcall program was effective in reducing unmet needs in caregivers. Provision of cancer information and support via a telephone service may represent a feasible approach to reducing unmet needs among cancer caregiver populations.

Trial registration: ACTRN12613000731796; prospectively registered on 02/07/2013.

Keywords: Telephone intervention, Caregivers, Cancer, RCT, Caregiver burden, Unmet needs, Depression, Health literacy, Helpline, Support

\footnotetext{
* Correspondence: I.heckel@deakin.edu.au

${ }^{1}$ Deakin University, Faculty of Health, School of Nursing and Midwifery,

Geelong, VIC 3220, Australia

Full list of author information is available at the end of the article
} 


\section{Background}

With the rise of cancer globally [1], the number of informal caregivers who provide uncompensated care to cancer patients is increasing proportionally [2]. In addition to their existing obligations, caregivers are often confronted with role transitions and new responsibilities of managing the needs of the person diagnosed with cancer [3, 4]. There is increasing recognition that informal caregivers of people with cancer need information and support from the health care system $[5,6]$. Failure to address these needs can impact substantially on caregivers' health causing considerable burden, anxiety, and depression [5, 7-10].

Since the Australian Clinical Practice Guidelines for Psychosocial Care of Adults with Cancer were introduced [11], no recommendations have been made for systematically supporting cancer caregivers. Although psychosocial interventions have been developed to support caregivers (e.g. [12-14]), a meta-analysis of 29 randomised controlled trials comprising psychoeducation, skills training, or therapeutic counselling interventions found only small to moderate effects but significantly reduced caregiver burden and improved aspects of quality of life [15]. Since then there have been calls for other tailored interventions to be designed and tested, particularly for those caring for patients in the early stages of the cancer trajectory $[16,17]$.

To address the gap in the literature, we linked a high quality and credible telephone-based information and support service (13 11 20) with caregivers who were caring for people recently diagnosed with cancer, in the early stages of treatment. Cancer Council's $13 \quad 1120$ Information and Support is a free telephone service providing tailored support to people affected by cancer across Australia. At present, individuals need to initiate contact with the 131120 service. The aim of this study was to evaluate whether a new model of service delivery (i.e. a telephone outcall program), provided by the established 131120 service, reduced caregiver burden and unmet needs, and improved psychological health among caregivers of people newly diagnosed with cancer.

\section{Methods}

\section{Research design}

This study was a single blind, multi-centre, randomised controlled trial. A detailed description of the study methods is available in the published protocol [18]. Briefly, patient/caregiver dyads were recruited at one private and three public health services in Melbourne and Adelaide, Australia, between August 2013 and December 2014. Eligible dyads were approached by trained research personnel at oncology outpatient units during treatment cycles 2-5 of adjuvant chemotherapy and/or fractions 2-10 of radiotherapy. A brief introduction to the trial was provided and interested dyads were given an information package to take home and followed up to confirm participation. Consenting dyads completed surveys at baseline and at 1 and 6 months postintervention.

\section{Participants}

Eligible patients were identified by nurses through hospital patient management systems and included those with a primary cancer diagnosis (any cancer type, stages I-III) who received treatment with curative intent. Minimum age of both patients and caregivers was 18 years and dyads had to be able to read and understand English language and present with no cognitive impairment to participate in the study. Caregivers were nominated by the patient as the person most involved in providing support throughout the illness trajectory.

\section{Cancer council 131120 information and support service}

Cancer Council Australia are the largest non-government provider of cancer support services (http://www.cancer.org.au/). Their signature service is the 131120 telephone information and support service, run by specialist oncology nurses with extensive clinical and counselling experience, which they use to educate, support and link callers to other internal and external services, depending on their needs.

\section{Randomisation and group allocation}

A computer-generated randomisation table stratified by health service was produced by the trial statistician and made accessible to the study co-ordinator who conducted the randomization. Dyads were randomised to the intervention or the attention control group and participants were blinded after group allocation. In the intervention group, caregivers received three calls from a 131120 nurse with the first outcall at the start of the program (5-10 days post-randomisation), the second outcall 1 month later and the last outcall 3 months following the second call. During each outcall, the nurse measured caregivers' distress using the Distress Thermometer [19] and offered referral to appropriate services to those with elevated scores (distress $\geq 4$ and impact $\geq 3$ ). The nurse then raised six topics for further discussion to address caregivers' potential unmet needs: psychological distress, health literacy, physical health, family support, financial burden, and practical difficulties (e.g. legal affairs). Caregivers could raise additional topics if required. In the attention control group, caregivers received three outcalls at the same time points as those in the intervention group (mean call duration: $3 \mathrm{~min}$, 22 min; respectively). These outcalls were conducted by trained research personnel who supplied caregivers with the 131120 number to self-initiate contact if needed, no other information or support was provided. 


\section{Measures}

Demographic characteristics were collected at baseline and included information on the caregiver (age, gender, postcode, type of relationship to the patient, living situation, household size, level of education) and patient (age, gender, postcode, treatment type, cancer diagnosis). Details of the measures used for caregiver and patient outcomes have been published [18]. Briefly, the primary outcome (caregiver burden) was measured using the Zarit Burden Interview (ZBI), which consists of 22 items [20]. A total score is calculated through summing individual item scores (range from 0 to 88 ) with higher scores indicating greater burden. Scores of 24 and above have been found to be indicative of risk for depression [21]. Secondary outcome measures for caregivers and patients were the Centre of Epidemiologic Studies - Depression scale (CES-D) [22] to measure depressive symptoms in caregivers and patients. This instrument consists of 20 items, each rated on a 4-point scale ranging from 'rarely or none of the time' to 'most or all of the time'. The Supportive Care Needs Survey for Partners \& Caregivers (SCNS-P\&C, 45 items) [23], and the Supportive Care Needs Survey (SCNS-SF34, 34 items) [24] were used to assess the perceived needs of caregivers and cancer patients respectively. Items on both tools are rated on a 5-point scale ranging from 'not applicable' to 'high need'. The Health Literacy Questionnaire (HLQ) [25] was used to assess caregivers' and patients' ability to obtain, understand and use health information. The HLQ consists of 44 items, each rated on a 5-point scale ranging from 'cannot do' to 'very easy'. The health education impact Questionnaire (heiQ) [26] was used to measure self-empowerment. The heiQ contains 40 items, each rated on a 4-point scale ranging from 'strongly disagree' to 'strongly agree'. The self-esteem subscale of the Caregiver Reaction Assessment (CRA, 7 items) was used to measure positive aspects of caregiving on a 5-point scale ranging from 'strongly disagree' to 'strongly agree' [27]. A self-designed utility assessment was included in the 1 month post-intervention survey to evaluate caregivers' perceptions of the outcall program (i.e. 'I feel it was worth my time and effort to take part in the outcall program').

\section{Sample size calculation}

The primary outcome was change in caregiver burden as measured by the ZBI. A sample size of 180 dyads (90 per group) at the end of the trial period was estimated to detect a moderate effect size $(d=0.5)$, which corresponds to a difference between the treatment groups of 6.3 units on the ZBI Total score, with $90 \%$ power and alpha $=0.05$ (two-sided).

\section{Data analysis}

An intention to treat analysis was performed and analyses were undertaken using GenStat and Stata. A mixed model analysis, using the restricted maximum likelihood method (REML), was used to calculate the between and within caregiver components of variance and the predicted main-effect means for study group, time (baseline and one-month post intervention) and the two-way interaction predicted means. A difference between the research groups in the change in caregiver burden was claimed if the F-test for the two-way interaction was significant $(P<0.05)$. Mixed model analyses were also used to analyse all secondary caregiver endpoints (CRA, CESD, heiQ domains, HLQ subscales). Data from the 45item SCNS-P\&C were summarised as the total number of (moderate/high) unmet needs [7, 23] and a variancestabilising square-root transformation was applied in mixed model analyses of this endpoint.

\section{Results \\ Caregiver and patient sample}

Overall, 839 patient/caregiver dyads were approached for study participation, of which 737 were eligible to participate and 216 (29\%) provided informed consent and were included in each analysis. Dyads were randomized into intervention $(n=108)$ and attention control $(n=108)$ groups (Fig. 1).

The overall attrition rate at 1 -month post intervention was $30 \%$ (intervention, $27 \%$; control, 32\%). Participants' demographic characteristics are provided in Table 1. At baseline, there were no significant differences between groups in participants' demographic or clinical characteristics.

\section{Caregiver outcomes}

Analyses of outcome variables for caregivers are summarized in Table 2 .

\section{Caregiver burden}

At baseline, the primary outcome variable caregiver burden (ZBI Total Score), in both the intervention group $(M=18.99, \mathrm{SE}=1.17)$ and the control group $(M=18.05$, $\mathrm{SE}=1.17)$ was low. The intervention had no significant effect on caregiver burden $(P=0.921)$. The estimated between-individual and within-individual variance components were 97.2 and 50.4 respectively $(\mathrm{ICC}=0.659)$.

\section{Caregiver unmet needs}

Unmet needs declined in both groups over the course of the intervention period $(P<0.001)$ and there was a significant interaction between study group and time $(P=0.023)$ with a greater decline from baseline to month 1 in the intervention group compared to the control group $(t=-2.703 ; \mathrm{df}=312.3 ; P=0.007)$. The 


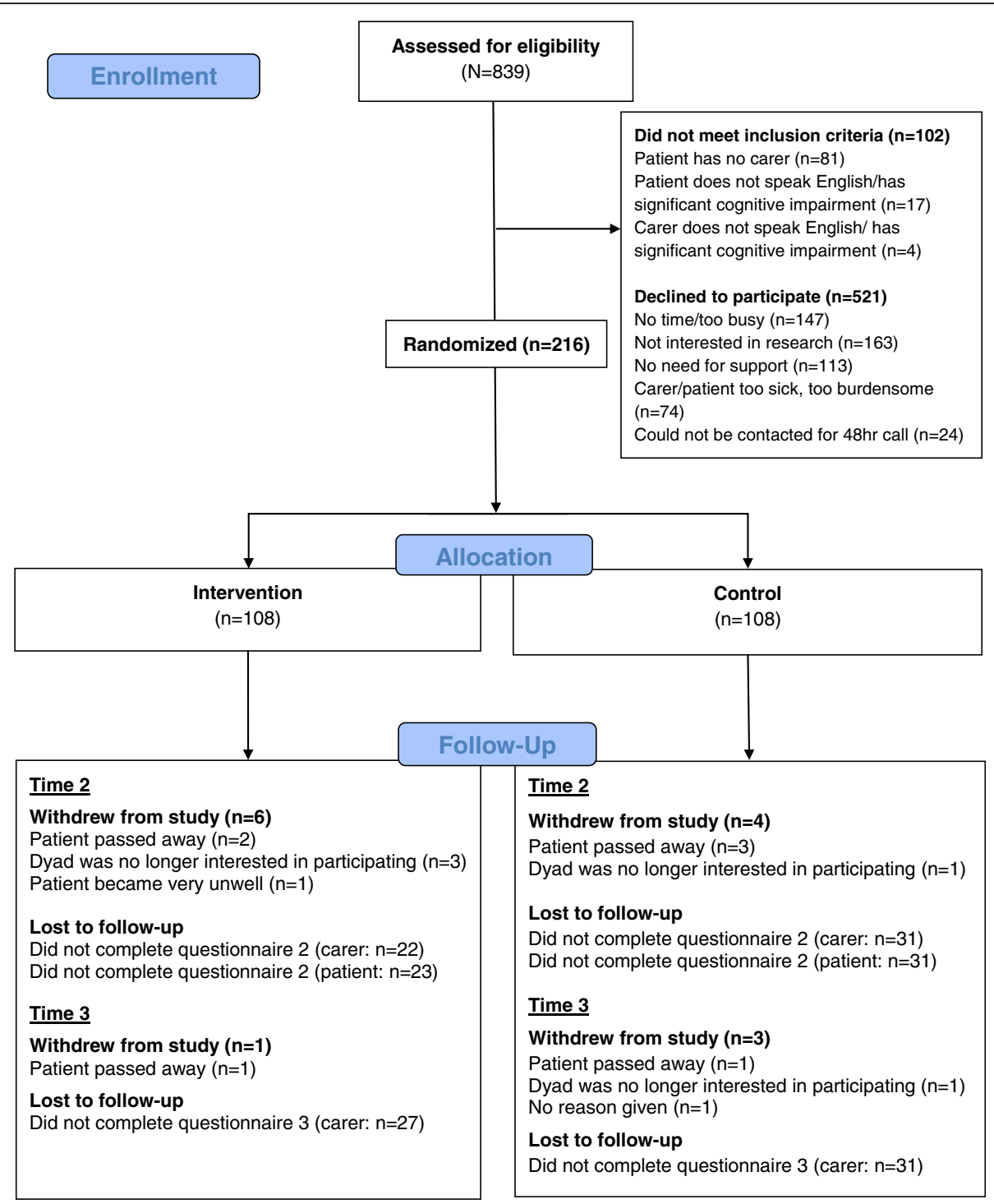

Fig. 1 Consort diagram showing recruitment of patient/caregiver dyads into the PROTECT study

decline from baseline to month 6 appeared to be greater in the intervention group but this was not significant at the conventional 5\% level $(t=-1.661$; df $=312.3 ; \quad P=0.098)$. Back-transformed means $(95 \%$ confidence intervals) at baseline, month 1 and month 6 were $1.30(0.80,1.94), 1.02(0.52,1.69)$ and 0.17 $(0.01,0.51)$ respectively in the control group, and $2.66(1.91,3.54), 0.85(0.42,1.44)$ and $0.28(0.06$, $0.68)$ respectively in the intervention group.

\section{Caregiver self-empowerment (heiQ) and health literacy}

There were no statistically significant differences between the study groups in their changes over time in heiQ subscales as indicated by the outcomes of F-tests for time by treatment interactions (Table 2). No significant effects were found for health literacy subscales (data not shown).

\section{Caregiver self-esteem}

Caregiver self-esteem (CRA) declined in both groups from baseline to months 1 and $6(P=0.045)$ and there was no significant difference between the groups in their declines over time $(P=0.320)$.

\section{Depressive symptoms and caregivers at risk for depression}

No significant effects were found for depressive symptoms (CES-D Total Score). A subsample of caregivers had burden scores of 24 or greater (intervention, $n=37$; control, $n=31$ ), which indicated risk for depression. Caregivers in these groups did not differ with respect to baseline demographic and clinical characteristics (Table 3). Significant demographic differences were found when comparing caregivers at risk for depression $(\mathrm{ZBI} \geq 24)$ with those not at risk $(\mathrm{ZBI}<24)$. Caregivers at 
Table 1 Baseline demographic and clinical characteristics of patients and caregivers in the PROTECT trial by study group $(N=216)$

\begin{tabular}{|c|c|c|c|}
\hline Characteristics & Control & Intervention & $P$ \\
\hline \multicolumn{4}{|l|}{ Patient demographics } \\
\hline Age, mean (SD), years & $\begin{array}{l}59.8 \\
(12.4)\end{array}$ & $58.8(12.1)$ & .58 \\
\hline Gender, n (\%) & & & .58 \\
\hline Male & $49(45.4)$ & $45(41.7)$ & \\
\hline Female & $59(54.6)$ & $63(58.3)$ & \\
\hline \multicolumn{4}{|l|}{ Clinical data } \\
\hline Type of Cancer, n (\%) & & & .21 \\
\hline Solid & $92(85.2)$ & $98(90.7)$ & \\
\hline Haematological & $16(14.8)$ & $10(9.3)$ & \\
\hline Treatment regimen, $\mathrm{n}(\%)$ & & & .85 \\
\hline Chemotherapy & $40(37.0)$ & $42(38.9)$ & \\
\hline Radiotherapy & $38(35.2)$ & $34(31.5)$ & \\
\hline Chemo/Radio combined & $30(27.8)$ & $32(29.6)$ & \\
\hline \multicolumn{4}{|l|}{ Caregiver demographics } \\
\hline Age, mean (SD), years & $\begin{array}{l}56.3 \\
(14.2)\end{array}$ & $57.2(11.6)$ & .59 \\
\hline \multicolumn{3}{|l|}{ Gender, n (\%) } & .27 \\
\hline Male & $42(38.9)$ & $50(46.3)$ & \\
\hline Female & $66(61.1)$ & $58(53.7)$ & \\
\hline \multicolumn{3}{|l|}{$\begin{array}{l}\text { Indigeneity- Aboriginal/Torres Strait } \\
\text { Islander, n (\%) }\end{array}$} & - \\
\hline Yes & $0(0)$ & $0(0)$ & \\
\hline No & $\begin{array}{l}104 \\
(100.0)\end{array}$ & $92(100.0)$ & \\
\hline \multicolumn{3}{|l|}{ Country of birth, n (\%) } & .46 \\
\hline Australia & $84(80.8)$ & $83(84.7)$ & \\
\hline Other & $20(19.2)$ & $15(15.3)$ & \\
\hline \multicolumn{3}{|l|}{ Lives with patient, $n(\%)$} & .85 \\
\hline Yes & $90(83.3)$ & $91(84.3)$ & \\
\hline No & $18(16.7)$ & $17(15.7)$ & \\
\hline \multicolumn{3}{|l|}{ Relationship to patient, n (\%) } & .87 \\
\hline Spouse/partner & $85(78.7)$ & $86(79.6)$ & \\
\hline Other (e.g. parent, adult child, friend) & $23(21.3)$ & $22(20.4)$ & \\
\hline \multicolumn{4}{|l|}{ Household size, mean (SD) } \\
\hline Number of people $\geq 18$ years & $2.2(0.7)$ & $2.2(0.7)$ & .85 \\
\hline Number of people $<18$ years & $0.4(0.9)$ & $0.4(0.9)$ & .88 \\
\hline Total household size & $2.6(1.1)$ & $2.6(1.2)$ & .81 \\
\hline \multicolumn{3}{|l|}{ Education status, n (\%) } & .85 \\
\hline Primary/secondary school & $48(44.9)$ & $47(43.5)$ & \\
\hline Certificate/Diploma & $27(25.2)$ & $25(23.1)$ & \\
\hline University Degree & $32(29.9)$ & $36(33.3)$ & \\
\hline
\end{tabular}

risk were about 6 years younger (52.9 vs 58.4 years; $P=0.003)$ and had more people in their households (2.9 vs 2.5 mean household size; $P=0.04$ ) than those not at risk (Table 4). For caregivers at risk, the intervention had a significant effect on having sufficient information to manage caregivers health $(P=0.040)$. Post hoc analyses showed an increase in caregivers' confidence that they had sufficient information to manage their health (HLQ Scale 2) between baseline and 6 months $(P=0.002)$ and between 1 month and 6 months $(P=0.009)$ in the intervention group but no such changes were observed in the control group $(P>0.30)$. No significant differences between the groups, in their changes over time, were observed for the other caregiver outcome variables.

For patients associated with caregivers at risk of depression, there was a significant decline in emotional distress from baseline (BL) to 1 month (M1) $(P=0.001)$. Investigation of the significant three-way interaction $(P=.027)$ indicated a significant decrease in emotional distress in the control group (BL mean $=2.592$, M1 mean $=2.046$, SED $=0.133, P<.001)$ but a non-significant decrease in the intervention group $(\mathrm{BL}$ mean $=2.487$, $\mathrm{M} 1$ mean $=$ $2.288, \mathrm{SED}=0.117, P=.089$ ). No other statistically significant effects were found.

\section{Patient outcomes}

No significant differences between the groups, in their changes over time were observed for patients' depressive symptoms, unmet needs or health literacy (Additional file 1: Table s1).

A significant improvement in positive and active engagement in life (heiQ, domain 2) among patients in the control group, but not the intervention group, was noted $(t=2.972 ; \mathrm{df}=168.4 ; \quad P=0.003)$. No significant effects were found for other subscales of the heiQ.

\section{Self-initiated contact to the 131120 service by caregivers in the attention control group}

Of the 108 participants in the attention control group, seven caregivers (6\%) initiated contact to the 131120 service between outcalls. Of those, two were repeat callers living in outer regional areas.

\section{Perceptions of caregivers on the outcall program}

Caregivers in the intervention group were asked in what way the outcall program had helped them in their role as a caregiver (Fig. 2). Most caregivers reported that the service had helped them to reduce their worries (74\%), to think positively about their situation (78\%), and to think things through (82\%). 
Table 2 -Time ( $\mathrm{BL}=$ Baseline, $\mathrm{M1}$ = Month 1, M6 = Month 6) by treatment means for outcome variables for caregiver

\begin{tabular}{|c|c|c|c|c|c|c|c|c|c|}
\hline \multirow[t]{2}{*}{ Outcome } & \multicolumn{3}{|c|}{ Control } & \multicolumn{3}{|c|}{ Intervention } & \multicolumn{3}{|c|}{$\Delta=$ Intervention - Control } \\
\hline & Mean & $\mathrm{SE}^{\mathrm{a}}$ & $\mathrm{n}$ (or $P$-value) $)^{\mathrm{b}}$ & Mean & SE & n (or $P$-value) & $\Delta$ & $\mathrm{SED}^{c}$ & $P \_$value \\
\hline \multicolumn{10}{|l|}{$\overline{\left.Z B\right|^{e}}$} \\
\hline$B L$ & 18.05 & 1.17 & 108 & 18.99 & 1.17 & 107 & 0.95 & 1.65 & 0.568 \\
\hline M1 & 17.81 & 1.31 & 73 & 19.39 & 1.27 & 82 & 1.58 & 1.82 & 0.386 \\
\hline M6 & 17.20 & 1.33 & 69 & 18.46 & 1.31 & 73 & 1.26 & 1.87 & 0.500 \\
\hline M1-BL & -0.24 & 1.13 & 0.835 & 0.40 & 1.09 & 0.713 & & & \\
\hline M6-BL & -0.85 & 1.16 & 0.464 & -0.53 & 1.14 & 0.639 & & & \\
\hline M6-M1 & -0.61 & 1.23 & 0.620 & -0.93 & 1.16 & 0.422 & & & $0.921^{d}$ \\
\hline \multicolumn{10}{|l|}{ CRA $^{f}$ Total } \\
\hline$B L$ & 30.07 & 0.44 & 108 & 30.80 & 0.44 & 105 & 0.73 & 0.62 & 0.243 \\
\hline M1 & 29.80 & 0.50 & 73 & 29.58 & 0.49 & 80 & -0.22 & 0.70 & 0.750 \\
\hline M6 & 29.76 & 0.52 & 66 & 29.66 & 0.51 & 70 & -0.09 & 0.73 & 0.897 \\
\hline M1-BL & -0.27 & 0.49 & 0.578 & -1.22 & 0.48 & 0.012 & & & \\
\hline M6-BL & -0.31 & 0.51 & 0.538 & -1.13 & 0.50 & 0.025 & & & \\
\hline M6-M1 & -0.04 & 0.54 & 0.940 & 0.09 & 0.52 & 0.867 & & & 0.320 \\
\hline \multicolumn{10}{|c|}{ SCNS-P\&C ${ }^{g}$ ( $\sqrt{ }$ transformed $)$} \\
\hline$B L$ & 1.14 & 0.13 & 108 & 1.63 & 0.13 & 108 & 0.49 & 0.18 & 0.007 \\
\hline M1 & 1.01 & 0.15 & 75 & 0.92 & 0.14 & 83 & -0.09 & 0.20 & 0.659 \\
\hline M6 & 0.41 & 0.15 & 68 & 0.53 & 0.15 & 73 & 0.12 & 0.21 & 0.577 \\
\hline M1-BL & -0.13 & 0.15 & 0.401 & -0.71 & 0.15 & $<0.001$ & & & \\
\hline M6-BL & -0.73 & 0.16 & $<0.001$ & -1.10 & 0.16 & $<0.001$ & & & \\
\hline M6-M1 & -0.60 & 0.17 & 0.001 & -0.39 & 0.16 & $<0.001$ & & & 0.023 \\
\hline \multicolumn{10}{|c|}{ CES-Dh Total } \\
\hline$B L$ & 11.46 & 0.91 & 107 & 13.24 & 0.91 & 108 & 1.79 & 1.29 & 0.168 \\
\hline M1 & 10.98 & 1.02 & 75 & 12.32 & 0.99 & 82 & 1.34 & 1.42 & 0.347 \\
\hline M6 & 10.62 & 1.04 & 68 & 12.04 & 1.02 & 73 & 1.42 & 1.46 & 0.334 \\
\hline M1-BL & -0.48 & 0.89 & 0.592 & -0.93 & 0.85 & 0.280 & & & \\
\hline M6-BL & -0.83 & 0.92 & 0.369 & -1.20 & 0.89 & 0.180 & & & \\
\hline M6-M1 & -0.36 & 0.97 & 0.715 & -0.27 & 0.92 & 0.765 & & & 0.926 \\
\hline \multicolumn{10}{|c|}{ heiQ' Dom1 HDB } \\
\hline$B L$ & 2.90 & 0.07 & 107 & 2.83 & 0.07 & 108 & -0.06 & 0.10 & 0.548 \\
\hline M1 & 3.05 & 0.08 & 75 & 2.81 & 0.08 & 82 & -0.24 & 0.11 & 0.034 \\
\hline M6 & 2.96 & 0.08 & 68 & 2.92 & 0.08 & 73 & -0.04 & 0.12 & 0.749 \\
\hline M1-BL & 0.15 & 0.07 & 0.026 & -0.03 & 0.07 & 0.680 & & & \\
\hline M6-BL & 0.06 & 0.07 & 0.379 & 0.09 & 0.07 & 0.202 & & & \\
\hline M6-M1 & -0.09 & 0.07 & 0.224 & 0.11 & 0.07 & 0.105 & & & 0.080 \\
\hline \multicolumn{10}{|c|}{ heiQ Dom2 PAEL } \\
\hline $\mathrm{BL}$ & 3.15 & 0.05 & 108 & 3.05 & 0.05 & 108 & -0.10 & 0.07 & 0.157 \\
\hline M1 & 3.18 & 0.06 & 75 & 3.02 & 0.05 & 82 & -0.17 & 0.08 & 0.035 \\
\hline M6 & 3.22 & 0.06 & 68 & 3.06 & 0.06 & 73 & -0.16 & 0.08 & 0.053 \\
\hline M1-BL & 0.03 & 0.05 & 0.464 & -0.03 & 0.05 & 0.509 & & & \\
\hline M6-BL & 0.07 & 0.05 & 0.166 & 0.01 & 0.05 & 0.782 & & & \\
\hline M6-M1 & 0.03 & 0.05 & 0.518 & 0.04 & 0.05 & 0.377 & & & 0.564 \\
\hline
\end{tabular}

heiQ Dom3 ED 
Table 2 -Time (BL = Baseline, M1 = Month 1, M6=Month 6) by treatment means for outcome variables for caregiver (Continued)

\begin{tabular}{|c|c|c|c|c|c|c|c|c|c|}
\hline \multirow[t]{2}{*}{ Outcome } & \multicolumn{3}{|c|}{ Control } & \multicolumn{3}{|c|}{ Intervention } & \multicolumn{3}{|c|}{$\Delta=$ Intervention - Control } \\
\hline & Mean & $\mathrm{SE}^{\mathrm{a}}$ & $\mathrm{n}(\text { or } P \text {-value })^{\mathrm{b}}$ & Mean & SE & n (or $P$-value) & $\Delta$ & $\mathrm{SED}^{c}$ & $P_{\text {_value }}$ \\
\hline$B L$ & 1.70 & 0.06 & 106 & 1.78 & 0.06 & 106 & 0.09 & 0.08 & 0.303 \\
\hline M1 & 1.73 & 0.07 & 75 & 1.95 & 0.06 & 82 & 0.22 & 0.09 & 0.015 \\
\hline M6 & 1.68 & 0.07 & 67 & 1.91 & 0.07 & 73 & 0.23 & 0.10 & 0.016 \\
\hline M1-BL & 0.03 & 0.06 & 0.653 & 0.17 & 0.06 & 0.005 & & & \\
\hline M6-BL & -0.02 & 0.06 & 0.766 & 0.13 & 0.06 & 0.036 & & & \\
\hline M6-M1 & -0.05 & 0.07 & 0.488 & -0.04 & 0.06 & 0.537 & & & 0.144 \\
\hline \multicolumn{10}{|c|}{ heiQ Dom4 SMl } \\
\hline$B L$ & 3.17 & 0.04 & 106 & 3.16 & 0.04 & 108 & -0.01 & 0.06 & 0.859 \\
\hline M1 & 3.13 & 0.05 & 75 & 3.07 & 0.04 & 82 & -0.06 & 0.06 & 0.354 \\
\hline M6 & 3.22 & 0.05 & 68 & 3.14 & 0.05 & 73 & -0.08 & 0.07 & 0.209 \\
\hline M1-BL & -0.04 & 0.04 & 0.418 & -0.08 & 0.04 & 0.047 & & & \\
\hline M6-BL & 0.06 & 0.05 & 0.199 & -0.01 & 0.04 & 0.749 & & & \\
\hline M6-M1 & 0.09 & 0.05 & 0.051 & 0.07 & 0.05 & 0.124 & & & 0.489 \\
\hline \multicolumn{10}{|c|}{ heiQ Dom5 CAA } \\
\hline$B L$ & 3.38 & 0.05 & 105 & 3.31 & 0.05 & 104 & -0.07 & 0.06 & 0.268 \\
\hline M1 & 3.30 & 0.05 & 75 & 3.20 & 0.05 & 82 & -0.11 & 0.07 & 0.124 \\
\hline M6 & 3.38 & 0.05 & 67 & 3.25 & 0.05 & 72 & -0.13 & 0.07 & 0.083 \\
\hline $\mathrm{M} 1-\mathrm{BL}$ & -0.08 & 0.05 & 0.117 & -0.12 & 0.05 & 0.016 & & & \\
\hline M6-BL & 0.00 & 0.05 & 0.980 & -0.06 & 0.05 & 0.256 & & & \\
\hline M6-M1 & 0.08 & 0.05 & 0.145 & 0.06 & 0.05 & 0.248 & & & 0.701 \\
\hline \multicolumn{10}{|c|}{ heiQ Dom6 STA } \\
\hline$B L$ & 3.17 & 0.04 & 102 & 3.15 & 0.04 & 103 & -0.02 & 0.06 & 0.794 \\
\hline M1 & 3.04 & 0.05 & 74 & 3.01 & 0.05 & 83 & -0.04 & 0.07 & 0.560 \\
\hline M6 & 3.17 & 0.05 & 66 & 3.09 & 0.05 & 73 & -0.08 & 0.07 & 0.262 \\
\hline M1-BL & -0.12 & 0.05 & 0.015 & -0.15 & 0.05 & 0.002 & & & \\
\hline M6-BL & 0.00 & 0.05 & 0.997 & -0.06 & 0.05 & 0.202 & & & \\
\hline M6-M1 & 0.12 & 0.05 & 0.026 & 0.08 & 0.05 & 0.108 & & & 0.672 \\
\hline \multicolumn{10}{|c|}{ heiQ Dom7 SIS } \\
\hline$B L$ & 3.23 & 0.05 & 106 & 3.12 & 0.05 & 104 & -0.11 & 0.07 & 0.114 \\
\hline M1 & 3.08 & 0.05 & 75 & 2.98 & 0.05 & 83 & -0.10 & 0.08 & 0.212 \\
\hline M6 & 3.24 & 0.06 & 68 & 3.05 & 0.06 & 73 & -0.19 & 0.08 & 0.016 \\
\hline M1-BL & -0.15 & 0.05 & 0.003 & -0.13 & 0.05 & 0.006 & & & \\
\hline M6-BL & 0.01 & 0.05 & 0.778 & -0.07 & 0.05 & 0.191 & & & \\
\hline M6-M1 & 0.16 & 0.05 & 0.003 & 0.07 & 0.05 & 0.193 & & & 0.392 \\
\hline \multicolumn{10}{|c|}{ heiQ Dom8 HSN } \\
\hline$B L$ & 3.27 & 0.05 & 105 & 3.17 & 0.05 & 103 & -0.10 & 0.07 & 0.153 \\
\hline M1 & 3.23 & 0.05 & 75 & 3.07 & 0.05 & 83 & -0.16 & 0.08 & 0.034 \\
\hline M6 & 3.39 & 0.06 & 67 & 3.15 & 0.05 & 73 & -0.24 & 0.08 & 0.003 \\
\hline M1-BL & -0.04 & 0.05 & 0.409 & -0.10 & 0.05 & 0.032 & & & \\
\hline M6-BL & 0.12 & 0.05 & 0.015 & -0.02 & 0.05 & 0.753 & & & \\
\hline M6-M1 & 0.16 & 0.05 & 0.002 & 0.09 & 0.05 & 0.086 & & & 0.144 \\
\hline \multicolumn{10}{|l|}{ HLQ' S1 } \\
\hline$B L$ & 3.24 & 0.05 & 105 & 3.11 & 0.05 & 103 & -0.13 & 0.07 & 0.083 \\
\hline
\end{tabular}


Table 2 -Time (BL = Baseline, M1 = Month 1, M6=Month 6) by treatment means for outcome variables for caregiver (Continued)

\begin{tabular}{|c|c|c|c|c|c|c|c|c|c|}
\hline \multirow[t]{2}{*}{ Outcome } & \multicolumn{3}{|c|}{ Control } & \multicolumn{3}{|c|}{ Intervention } & \multicolumn{3}{|c|}{$\Delta=$ Intervention - Control } \\
\hline & Mean & $S E^{a}$ & $\mathrm{n}(\text { or } P \text {-value) })^{\mathrm{b}}$ & Mean & SE & $\mathrm{n}$ (or $P$-value) & $\Delta$ & $\mathrm{SED}^{c}$ & P_value \\
\hline M1 & 3.25 & 0.06 & 73 & 3.15 & 0.06 & 83 & -0.10 & 0.08 & 0.208 \\
\hline M6 & 3.30 & 0.06 & 67 & 3.17 & 0.06 & 73 & -0.13 & 0.08 & 0.109 \\
\hline M1-BL & 0.01 & 0.05 & 0.913 & 0.03 & 0.05 & 0.492 & & & \\
\hline M6-BL & 0.06 & 0.05 & 0.220 & 0.06 & 0.05 & 0.239 & & & \\
\hline M6-M1 & 0.06 & 0.05 & 0.288 & 0.03 & 0.05 & 0.604 & & & 0.892 \\
\hline \multicolumn{10}{|l|}{ HLQ S2 } \\
\hline $\mathrm{BL}$ & 3.15 & 0.04 & 106 & 3.10 & 0.04 & 105 & -0.05 & 0.06 & 0.388 \\
\hline M1 & 3.15 & 0.05 & 74 & 3.09 & 0.05 & 83 & -0.06 & 0.07 & 0.392 \\
\hline M6 & 3.23 & 0.05 & 67 & 3.19 & 0.05 & 73 & -0.04 & 0.07 & 0.552 \\
\hline M1-BL & -0.01 & 0.05 & 0.880 & -0.01 & 0.04 & 0.785 & & & \\
\hline M6-BL & 0.08 & 0.05 & 0.107 & 0.09 & 0.05 & 0.058 & & & \\
\hline M6-M1 & 0.08 & 0.05 & 0.097 & 0.10 & 0.05 & 0.034 & & & 0.970 \\
\hline \multicolumn{10}{|l|}{ HLQ S3 } \\
\hline $\mathrm{BL}$ & 2.96 & 0.05 & 107 & 2.89 & 0.05 & 105 & -0.07 & 0.07 & 0.282 \\
\hline $\mathrm{M} 1$ & 2.95 & 0.05 & 74 & 2.91 & 0.05 & 83 & -0.04 & 0.07 & 0.616 \\
\hline M6 & 2.99 & 0.05 & 67 & 2.91 & 0.05 & 72 & -0.08 & 0.08 & 0.286 \\
\hline M1-BL & -0.02 & 0.05 & 0.758 & 0.02 & 0.05 & 0.692 & & & \\
\hline M6-BL & 0.03 & 0.05 & 0.558 & 0.02 & 0.05 & 0.695 & & & \\
\hline M6-M1 & 0.05 & 0.05 & 0.405 & 0.00 & 0.05 & 0.987 & & & 0.815 \\
\hline \multicolumn{10}{|l|}{ HLQ S4 } \\
\hline BL & 3.19 & 0.05 & 106 & 3.04 & 0.05 & 105 & -0.16 & 0.07 & 0.019 \\
\hline M1 & 3.12 & 0.05 & 74 & 3.02 & 0.05 & 83 & -0.10 & 0.07 & 0.166 \\
\hline M6 & 3.23 & 0.05 & 68 & 3.04 & 0.05 & 73 & -0.19 & 0.07 & 0.010 \\
\hline M1-BL & -0.07 & 0.05 & 0.119 & -0.02 & 0.04 & 0.721 & & & \\
\hline M6-BL & 0.04 & 0.05 & 0.382 & 0.00 & 0.05 & 0.940 & & & \\
\hline M6-M1 & 0.11 & 0.05 & 0.024 & 0.02 & 0.05 & 0.682 & & & 0.381 \\
\hline \multicolumn{10}{|l|}{ HLQ S5 } \\
\hline $\mathrm{BL}$ & 2.90 & 0.05 & 104 & 2.86 & 0.05 & 104 & -0.04 & 0.07 & 0.587 \\
\hline M1 & 2.89 & 0.06 & 73 & 2.88 & 0.05 & 83 & -0.01 & 0.08 & 0.878 \\
\hline M6 & 2.95 & 0.06 & 67 & 2.91 & 0.06 & 73 & -0.04 & 0.08 & 0.587 \\
\hline M1-BL & -0.01 & 0.05 & 0.802 & 0.01 & 0.05 & 0.788 & & & \\
\hline M6-BL & 0.05 & 0.05 & 0.372 & 0.04 & 0.05 & 0.412 & & & \\
\hline M6-M1 & 0.06 & 0.06 & 0.282 & 0.03 & 0.05 & 0.578 & & & 0.903 \\
\hline \multicolumn{10}{|l|}{ HLQ S6 } \\
\hline$B L$ & 4.22 & 0.06 & 106 & 4.23 & 0.06 & 104 & 0.01 & 0.09 & 0.863 \\
\hline M1 & 4.19 & 0.07 & 74 & 4.28 & 0.07 & 83 & 0.10 & 0.10 & 0.322 \\
\hline M6 & 4.28 & 0.07 & 68 & 4.18 & 0.07 & 73 & -0.10 & 0.10 & 0.331 \\
\hline M1-BL & -0.03 & 0.07 & 0.626 & 0.05 & 0.07 & 0.470 & & & \\
\hline M6-BL & 0.06 & 0.07 & 0.388 & -0.05 & 0.07 & 0.454 & & & \\
\hline M6-M1 & 0.09 & 0.07 & 0.207 & -0.10 & 0.07 & 0.158 & & & 0.168 \\
\hline \multicolumn{10}{|l|}{ HLQ S7 } \\
\hline$B L$ & 4.14 & 0.06 & 104 & 4.12 & 0.06 & 104 & -0.03 & 0.09 & 0.772 \\
\hline M1 & 4.10 & 0.07 & 73 & 4.21 & 0.07 & 83 & 0.11 & 0.10 & 0.259 \\
\hline
\end{tabular}


Table 2 -Time (BL = Baseline, M1 = Month 1, M6= Month 6) by treatment means for outcome variables for caregiver (Continued)

\begin{tabular}{|c|c|c|c|c|c|c|c|c|c|}
\hline \multirow[t]{2}{*}{ Outcome } & \multicolumn{3}{|c|}{ Control } & \multicolumn{3}{|c|}{ Intervention } & \multicolumn{3}{|c|}{$\Delta=$ Intervention - Control } \\
\hline & Mean & $\mathrm{SE}^{\mathrm{a}}$ & $\mathrm{n}\left(\right.$ or $P$-value) ${ }^{\mathrm{b}}$ & Mean & SE & $\mathrm{n}$ (or $P$-value) & $\Delta$ & $\mathrm{SED}^{c}$ & $P_{\text {_value }}$ \\
\hline M6 & 4.19 & 0.07 & 68 & 4.14 & 0.07 & 73 & -0.05 & 0.10 & 0.629 \\
\hline M1-BL & -0.04 & 0.06 & 0.498 & 0.09 & 0.06 & 0.143 & & & \\
\hline M6-BL & 0.05 & 0.07 & 0.488 & 0.02 & 0.06 & 0.717 & & & \\
\hline M6-M1 & 0.09 & 0.07 & 0.200 & -0.07 & 0.06 & 0.306 & & & 0.192 \\
\hline \multicolumn{10}{|l|}{ HLQ S8 } \\
\hline$B L$ & 4.15 & 0.06 & 105 & 4.13 & 0.06 & 104 & -0.03 & 0.09 & 0.774 \\
\hline M1 & 4.08 & 0.07 & 73 & 4.18 & 0.07 & 83 & 0.09 & 0.10 & 0.341 \\
\hline M6 & 4.19 & 0.07 & 68 & 4.13 & 0.07 & 73 & -0.05 & 0.10 & 0.614 \\
\hline M1-BL & -0.07 & 0.07 & 0.333 & 0.05 & 0.07 & 0.473 & & & \\
\hline M6-BL & 0.03 & 0.07 & 0.657 & 0.01 & 0.07 & 0.928 & & & \\
\hline M6-M1 & 0.10 & 0.08 & 0.192 & -0.04 & 0.07 & 0.559 & & & 0.336 \\
\hline \multicolumn{10}{|l|}{ HLQ S9 } \\
\hline$B L$ & 4.31 & 0.05 & 104 & 4.30 & 0.05 & 104 & -0.01 & 0.08 & 0.916 \\
\hline M1 & 4.27 & 0.06 & 74 & 4.37 & 0.06 & 83 & 0.10 & 0.08 & 0.234 \\
\hline M6 & 4.32 & 0.06 & 68 & 4.37 & 0.06 & 73 & 0.05 & 0.09 & 0.563 \\
\hline M1-BL & -0.04 & 0.06 & 0.512 & 0.07 & 0.06 & 0.197 & & & \\
\hline M6-BL & 0.00 & 0.06 & 0.948 & 0.06 & 0.06 & 0.279 & & & \\
\hline M6-M1 & 0.04 & 0.06 & 0.504 & -0.01 & 0.06 & 0.884 & & & 0.390 \\
\hline
\end{tabular}

a SE Standard Error of the Mean or a difference in Time Means

b Sample sizes ( $n$ ) for means and $p$-values for time differences within treatment groups

c SED Standard Error of the Difference in Treatment group means within a Time

${ }^{d} \mathrm{P}$-value for the 2 degree-of-freedom test for a Time by Treatment group interaction

e ZBI Zarit Burden Interview

${ }^{f}$ CRA Caregiver Reaction Assessment

g SCNS - P\&C Supportive Care Needs Survey - Partner \& Caregivers

${ }^{\mathrm{h}}$ CES-D Centre for Epidemiologic Studies - Depression

' heiQ health education impact Questionnaire: Domain 1 - health directed behaviour (HDB), Domain 2 - positive and active engagement in life (PAEL), Domain 3 emotional distress (ED), Domain 4 - self monitoring and insight (SMI), Domain 5 - constructive attitudes and approaches (CAA), Domain 6 - skills and technique acquisition (STA), Domain 7 - social integration and support (SIS), Domain 8 - health service navigation (HSN)

${ }^{j}$ HLQ Health Literacy Questionnaire: S1 - feeling understood and supported by healthcare providers, S2 - having sufficient information to manage my health, S3 actively managing my health, S4 - social support for health, S5 - appraisal of health information, S6 - ability to actively engage with health care providers, S7 navigating the health care system, S8 - ability to find good health information, S9 - understanding health information well enough to know what to do

\section{Discussion}

This study tested the impact of a telephone outcall intervention delivered by two Australian Cancer Council 1311 20 services on the psychological distress of caregivers of newly diagnosed cancer patients. Results indicated that the outcall program did not reduce caregivers' overall caregiver burden. This may be due to the inclusion of caregivers with various levels of burden in this study and the fact that burden levels at baseline, for the majority of caregivers, were reasonably low, limiting the detection of changes over time.

While the outcall program had no significant impact on depressive symptoms in caregivers, it was associated with an increase in health literacy in a subgroup of caregivers who were at increased risk for depression at baseline (ZBI scores of $\geq 24$ ). For these caregivers, the intervention significantly increased caregivers' confidence in having sufficient information to manage their own (as opposed to the patient's) health. It is possible that these caregivers had low health literacy, hence the high burden levels, and the informational component of the intervention was able to meet caregivers' needs in this domain. We also found that caregivers 'at risk' were those who were younger in age and had more people residing in their households than those not at risk. Since this subgroup was relatively small (intervention, $n=37$; control, $n=31$ ) our ability to detect a significant impact, if in fact it exists, of the outcall program was impaired. Future research may consider screening caregivers for age, household size, and associated burden such as the care of young children or employment, as those may benefit from interventions of this type.

The outcall program produced positive changes over time with a greater reduction in the number of caregivers' reported unmet needs. The six topics raised for discussion by the 131120 nurses at each outcall were specifically chosen to address caregiver's supportive care 
Table 3 -Baseline demographic and clinical characteristics of patients and caregivers in the PROTECT trial by study group: Dyads including caregivers at risk of depression $(n=68)$

\begin{tabular}{|c|c|c|c|}
\hline Characteristics & Control & Intervention & $P$ \\
\hline \multicolumn{4}{|l|}{ Patient demographics } \\
\hline Age, mean (SD), years & $\begin{array}{l}59.1 \\
(12.9)\end{array}$ & $57.7(11.6)$ & .63 \\
\hline Gender, n (\%) & & & .81 \\
\hline Male & $15(48.4)$ & $19(51.4)$ & \\
\hline Female & $16(51.6)$ & $18(48.6)$ & \\
\hline \multicolumn{4}{|l|}{ Clinical data } \\
\hline Type of Cancer, n (\%) & & & .09 \\
\hline Solid & $24(77.4)$ & $34(91.9)$ & \\
\hline Haematological & $7(22.6)$ & $3(8.1)$ & \\
\hline Treatment regimen, n (\%) & & & .17 \\
\hline Chemotherapy & $14(45.2)$ & $13(35.1)$ & \\
\hline Radiotherapy & $11(35.5)$ & $9(24.3)$ & \\
\hline Chemo/Radio combined & $6(19.4)$ & $15(40.5)$ & \\
\hline \multicolumn{4}{|l|}{ Caregiver demographics } \\
\hline Age, mean (SD), years & $\begin{array}{l}53.6 \\
(12.9)\end{array}$ & $52.3(12.3)$ & .67 \\
\hline Gender, n (\%) & & & .67 \\
\hline Male & $11(35.5)$ & $15(40.5)$ & \\
\hline Female & $20(64.5)$ & $22(59.5)$ & \\
\hline $\begin{array}{l}\text { Indigeneity- Aboriginal/Torres Strait Islander, } \\
\mathrm{n}(\%)\end{array}$ & & & - \\
\hline Yes & $0(0)$ & $0(0)$ & \\
\hline No & $\begin{array}{l}31 \\
(100.0)\end{array}$ & $37(100.0)$ & \\
\hline Country of birth, n (\%) & & & .21 \\
\hline Australia & $23(79.3)$ & 29 (90.6) & \\
\hline Other & $6(20.7)$ & $3(9.4)$ & \\
\hline Lives with patient, $\mathrm{n}(\%)$ & & & .52 \\
\hline Yes & $26(83.9)$ & $33(89.2)$ & \\
\hline No & $5(16.1)$ & $4(10.8)$ & \\
\hline Relationship to patient, n (\%) & & & .33 \\
\hline Spouse/partner & $23(74.2)$ & $31(83.8)$ & \\
\hline Other (e.g. parent, adult child, friend) & $8(25.8)$ & $6(16.2)$ & \\
\hline \multicolumn{4}{|l|}{ Household size, mean (SD) } \\
\hline Number of people $\geq 18$ years & $2.3(0.8)$ & $2.2(0.6)$ & .65 \\
\hline Number of people $<18$ years & $0.6(1.0)$ & $0.6(1.2)$ & .97 \\
\hline Total household size & $2.9(1.3)$ & $2.8(1.3)$ & .84 \\
\hline Education status, n (\%) & & & .99 \\
\hline Primary/secondary school & $11(36.7)$ & $13(35.1)$ & \\
\hline Certificate/Diploma & $10(33.3)$ & $13(35.1)$ & \\
\hline University Degree & $9(30.0)$ & $11(39.7)$ & \\
\hline
\end{tabular}

Table 4 Baseline demographic and clinical characteristics of patients and caregivers in the PROTECT trial: Comparison of dyads including caregivers at risk of depression with those not at risk $\left(n=215^{\mathrm{a}}\right)$

\begin{tabular}{lll}
\hline Characteristics & $\begin{array}{l}\text { Caregivers not at } \\
\text { risk }(n=147)\end{array}$ & $\begin{array}{l}\text { Caregivers at } \\
\text { risk }(n=68)\end{array}$ \\
\hline $\begin{array}{l}\text { Patient demographics } \\
\text { Age, mean (SD), years }\end{array}$ & $59.6(12.3)$ & $58.3(12.1)$ \\
$\begin{array}{l}\text { Gender, n (\%) } \\
\text { Male }\end{array}$ & $59(40.1)$ & $34(50.0)$ \\
$\quad$ Female & $88(59.9)$ & $34(50.0)$
\end{tabular}

Clinical data

Type of Cancer, n (\%)

Solid

Haematological

Treatment regimen, $\mathrm{n}(\%)$

Chemotherapy

$55(37.4)$

Radiotherapy

Chemo/Radio combined

$51(34.7)$

Caregiver demographics

Age, mean (SD), years

Gender, n (\%)

Male

Female

$81(55.1)$

Indigeneity- Aboriginal/Torres

Strait Islander, $\mathrm{n}(\%)$

Yes

No

Country of birth, $\mathrm{n}(\%)$

Australia

Other

$26(18.6)$

Lives with patient, $\mathrm{n}(\%)$

$$
\text { Yes }
$$

No

$26(17.7)$

Relationship to patient, n (\%)

\section{Spouse/partner}

Other

(i.e. parent, adult child, friend)

Household size, mean (SD)

Number of people $\geq 18$ years

Number of people $<18$ years

Total household size

Education status, n (\%)

Primary/secondary school

Certificate/Diploma

University Degree
$131(89.1) \quad 58(85.3)$

$16(10.9)$

$10(14.7)$

$27(39.7)$

$20(29.4)$

41 (27.9)

$21(30.9)$

$58.4(12.8)$

$52.9(12.5) \quad .003$

66 (44.9)

.36

$26(38.2)$

$0(0)$

$0(0)$

$147(100.0)$

$68(100.0)$

$114(81.4)$

$52(85.2)$

$9(14.8)$

$59(86.8)$

.42

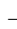

.51

.41

.93

$116(78.9) \quad 54(79.4)$

$31(21.1) \quad 14(20.6)$

$2.2(0.8) \quad 2.3(0.7) \quad .35$

$0.4(0.7) \quad 0.6(1.1) \quad .10$

$\begin{array}{lll}2.5(1.0) & 2.9(1.3) \quad .04\end{array}$

.06

$70(47.6) \quad 24(35.8)$

$29(19.7) \quad 23(34.3)$

$48(32.7) \quad 20(29.9)$ 


\section{Did the service...}

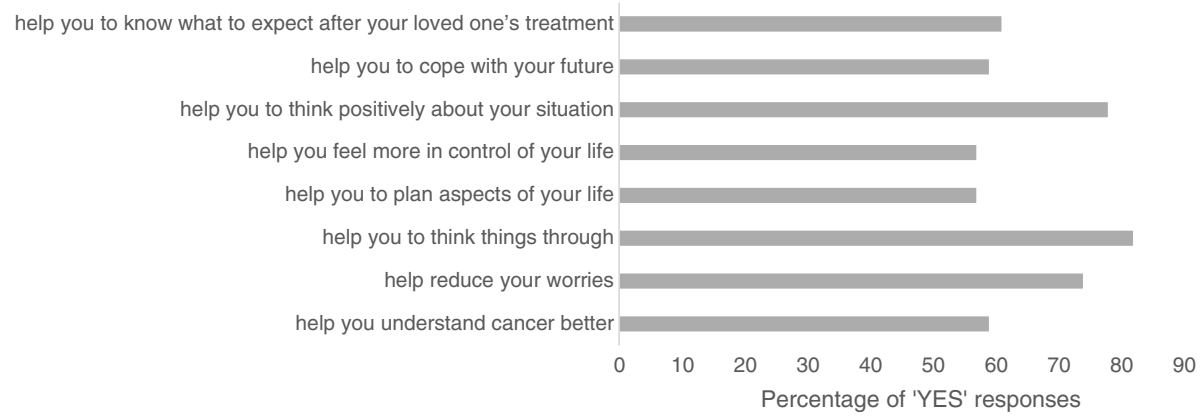

Fig. 2 Caregivers' perception of the value of the telephone outcall program

needs previously reported in the literature [4]. Researchers have stressed the importance of targeting caregivers' needs to minimise long-term health problems $[5,8,9]$. Therefore, our program, which addressed caregivers' unique needs at the early stages of the cancer trajectory, may be a good approach in preventing longterm negative health outcomes in caregivers. This hypothesis warrants further investigation.

We hypothesised that improved caregiver outcomes would impact positively on patient health and wellbeing. However, the outcall program had no significant effect on patient outcomes and our subgroup of burdened caregivers $(\mathrm{ZBI}>24)$ was possibly underpowered to detect significant improvements in patient outcomes. Targeting dyad interventions to specific subgroups (e.g. significantly burdened caregivers) may be more effective in improving patient wellbeing, however more research is needed to support this argument.

The outcall program was reported as beneficial by trial participants. The majority of caregivers stated that the outcalls helped them to reduce their worries and think more positively about their situation. These findings suggest that the program was acceptable to caregivers and may represent a feasible approach to provide information and support to a targeted population.

Limitations of this study included a modest recruitment rate of $29 \%$, which reflects the difficulty of enrolling cancer dyads into randomised trials consistently reported in the literature [28-31]. It is possible that eligible dyads who experienced significant burden declined participation due to the perceived burden of taking part in research, even though the intervention may have been of benefit to them. The overall attrition rate $(30 \%)$ in this study at month 1 was higher than the estimated attrition rate of $20 \%$. Further, we cannot rule out that caregivers in the attention control group actively sought support elsewhere as their unmet needs also declined from baseline to month 1 . Despite these limitations, the findings suggest that access to support services for caregivers, who are at risk for depression, can ameliorate the demands of caregiving and potentially improve quality of life outcomes; telephone information support services are a feasible approach to providing such access and warrant further investigation in this high risk group.

\section{Conclusion}

While the 4-month telephone outcall program did not reduce caregiver burden, it was effective in reducing caregivers' reported unmet supportive care needs. One third of caregivers were found to be at risk of depression and this was particularity the case in younger caregivers and those residing in larger households. Findings suggest that the provision of cancer information and support via a telephone service may represent a feasible approach to address caregivers' unique needs at the early stages of the cancer trajectory. However, the role of a telephone service to help prevent medium- and long-term negative health outcomes in this population group warrants further research.

\section{Additional file}

Additional file 1: Table S1. Patient outcomes. (DOCX 14 kb)

\begin{abstract}
Abbreviations
ANZCTR: Australian New Zealand Clinical Trials Registry; BL: Baseline; CESD: Centre of Epidemiologic Studies - Depression scale; Cl: Confidence Interval; CRA: Caregiver Reaction Assessment; df: Degree of freedom; heiQ: Health education impact Questionnaire; HLQ: Health Literacy Questionnaire; M: Mean; M1: 1 Month; M6 : 6 Month; RCT: Randomized Controlled Trial; REML: Restricted Maximum Likelihood Method; SCNSP\&C: Supportive Care Needs Survey for Partners \& Caregivers; SCNSSF34: Supportive Care Needs Survey; SD: Standard deviation; SE: Standard Error; SED: Standard Error of the Difference; ZBI: Zarit Burden Interview
\end{abstract}

\section{Acknowledgements}

We gratefully acknowledge our participants, the four health services (Epworth HealthCare, Eastern Health, Barwon Health, and the Royal Adelaide Hospital), the 131120 nurses at the two Cancer Councils (Chris Rivett, Jo White, Benita Heritage, Monica Conway, Valerie O'Mahony, Robyn Tucker), and all recruitment staff for their support and involvement in this study. 


\section{Funding}

This study was funded by the National Health and Medical Research Council (ID: GNT1044400RM24525). The funding source had no involvement in the design of the study, in the collection, analysis and interpretation of the data. The source was not involved in the preparation, writing and review of the manuscript or the decision to submit the manuscript for publication.

\section{Availability of data and materials}

The dataset used and/or analysed during the current study are available from the corresponding author on reasonable request.

\section{Authors' contributions}

$J R, A B, M B, R H O, C M, J C, M W, D M A$, and PML contributed to the design and conception of the study. $\mathrm{LH}, \mathrm{KMF}$ and $\mathrm{AB}$ were involved in the data acquisition and quality control of data. JR and CG conducted the statistical analysis; LH, JR, CG, and PML prepared and drafted the manuscript; LH revised the manuscript. All authors were involved in result interpretation, reviewed and approved the final manuscript

\section{Ethics approval and consent to participate}

This study was approved by Deakin University Human Research Ethics Committee (reference: 2012-083) and the respective committees at each participating health service and Cancer Council. All participants have given their written informed consent.

\section{Consent for publication}

Not applicable.

\section{Competing interests}

The authors declare that they have no competing interests.

\section{Publisher's Note}

Springer Nature remains neutral with regard to jurisdictional claims in published maps and institutional affiliations.

\section{Author details}

'Deakin University, Faculty of Health, School of Nursing and Midwifery, Geelong, VIC 3220, Australia. ²Deakin University, Faculty of Health, School of Health and Social Development, Geelong, VIC 3220, Australia. ${ }^{3}$ Cancer Council SA, 202 Greenhill Road, East wood, South Australia 5063, Australia. ${ }^{4}$ Flinders Centre for Innovation in Cancer, School of Medicine, Flinders University, Sturt Road, Bedford Park, South Australia 5042, Australia. ${ }^{5}$ Sansom Institute for Health Research, University of South Australia, City East Campus, North Terrace, Adelaide, South Australia, Australia. ${ }^{6}$ Monash University, Faculty of Medicine, Nursing and Health Sciences, Clayton, VIC 3168 Australia. ${ }^{7}$ Cancer Council Victoria, 615 St Kilda Road, Melbourne, VIC 3004, Australia. ${ }^{8}$ The University of Melbourne, Grattan Street, Parkville, VIC 3052 Australia. ${ }^{9}$ Epworth HealthCare, Richmond, VIC 3121, Australia. ${ }^{10}$ Eastern Health, Department of Oncology, Box Hill, VIC 3128, Australia. ${ }^{11}$ University of Newcastle, Faculty of Health and Medicine, Callaghan, NSW 2308, Australia. ${ }^{12}$ Barwon South Western Regional Integrated Cancer Service, Geelong, VIC 3220, Australia. ${ }^{13}$ Deakin University, Faculty of Health, Biostatistics Unit, Geelong, VIC 3220, Australia. ${ }^{14}$ The Andrew Love Cancer Centre, Geelong, VIC 3220, Australia.

Received: 9 August 2017 Accepted: 21 December 2017

Published online: 08 January 2018

\section{References}

1. Global Burden of Disease Cancer Collaboration, Fitzmaurice C, Dicker D, Pain A, Hamavid H, Moradi-Lakeh M, et al. The global burden of cancer 2013. JAMA Oncol. 2015;1:505-27.

2. Kent EE, Rowland JH, Northouse L, Litzelman K, Chou WS, Shelburne N, Timura C, O'Mara A, Huss K. Caring for caregivers and patients: research and clinical priorities for informal cancer caregiving. Cancer. 2016:122:1987-95.

3. Girgis A, Lambert SD. Caregivers of cancer survivors: the state of the field. Cancer Forum. 2009:33:167-71.

4. Lambert SD, Harrison JD, Smith E, Bonevski B, Carey M, Lawsin C, Paul C, Girgis A. The unmet needs of partners and caregivers of adults diagnosed with cancer: a systematic review. BMJ Suppor Palliat Care. 2012;2:224-30.
5. Girgis A, Lambert S, Johnson C, Waller A, Currow D. Physical, psychosocial, relationship, and economic burden of caring for people with cancer: a review. J Oncol Practice. 2013;9:197-202.

6. Girgis A, Lambert SD, McElduff P, Bonevski B, Lecathelinais C, Boyes A, Stacey F. Some things change, some things stay the same: a longitudinal analysis of cancer caregivers' unmet supportive care needs. Psycho-Oncology. 2013:22:1557-64

7. Heckel L, Fennell KM, Reynolds J, Osborne RH, Chirgwin J, Botti M, Ashley DM, Livingston PM. Unmet needs and depression among carers of people newly diagnosed with cancer. EJC. 2015;51:2049-57.

8. Jaafar NRN, Din SHS, Saini SM, Ahmad SNA, Midin M, Sidi H, Silim UA, Baharudin A. Clinical depression while caring for loved ones with breast cancer. Compr Psychiatry. 2014;55(Suppl):52-9.

9. Lee YH, Liao YC, Liao WY, Shun SC, Liu YC, Chan JC, Yu CJ, Yang PC, Lai $\mathrm{YH}$. Anxiety, depression and related factors in family caregivers of newly diagnosed lung cancer patients before first treatment. Psych-oncology. 2013:22:2617-23.

10. O'Toole MS, Zachariae R, Renna ME, Mennin DS, Applebaum A. Cognitive behavioral therapies for informal caregivers of patients with cancer and cancer survivors: a systematic review and meta analysis. Psychooncology. 2017;26(4):428-37.

11. National Breast Cancer Centre (NBCC) \& National Cancer Control Initiative (NCCl). Clinical practice guidelines for the psychosocial care of adults with cancer. Camperdown, NSW: National Breast Cancer Centre; 2003.

12. Badger T, Segrin C, Dorros SM, Meek P, Lopez AM. Depression and anxiety in women with breast cancer and their partners. Nurs Res. 2007;56:44-53.

13. Budin WC, Hoskins CN, Haber J, Sherman DW, Maislin G, Cater JR, et al. Breast cancer: education, counseling, and adjustment among patients and partners: a randomized clinical trial. Nurs Res. 2008:57:199-213.

14. Shum NF, Lui YL, Law WL, Fong YTD. A nurse-led psycho-education programme for Chinese carers of patients with colorectal cancer. Cancer Nurs Pract. 2014;13(5):31-9.

15. Northouse LL, Katapodi MC, Song L, Zhang L, Mood DW. Interventions with family caregivers of cancer patients, meta-analysis of randomized trials. CA: A Cancer J Clin. 2010:60:317-39.

16. Romito F, Goldzweig G, Cormio C, Hagedoorn M, Andersen BL. Informal caregiving for cancer patients. Cancer. 2013;119:2160-9.

17. Waldron EA, Janke EA, Bechtel CF, Ramirez M, Cohen A. A systematic review of psychosocial interventions to improve cancer caregiver quality of life. Psycho-Oncology. 2013;22(6):1200-7.

18. Livingston PM, Osborne RH, Botti M, Mihalopoulos C, McGuigan S, Heckel L Gunn K, Chirgwin J, Ashley DM, Williams M. Efficacy and cost-effectiveness of an outcall program to reduce carer burden and depression among carers of cancer patients [PROTECT]: rationale and design of a randomized controlled trial. BMC Health Serv Res. 2014;14:1-8.

19. National Comprehensive Cancer Network. Distress management. Clinical practice guidelines. J Natl Compr Cancer Netw. 2003;1:344-74.

20. Zarit SH, Reever KE, Bach-Peterson J. Relatives of the impaired elderly: correlates of feelings of burden. Gerontologist. 1980;20:649-55.

21. Schreiner AS, Morimoto T, Arai Y, Zarit S. Assessing family caregiver's mental health using a statistically derived cut-off score for the Zarit burden interview. Aging Ment Health. 2006:10:107-11.

22. Radloff LS. The CES-D scale: a self-report depression scale for research in the general population. Appl Psychol Meas. 1977:1:385-401.

23. Girgis A, Lambert S, Lecathelinais C. The supportive care needs survey for partners and caregivers of cancer survivors: development and psychometric evaluation. Psycho-Oncology. 2011;20:387-93.

24. Boyes A, Girgis A, Lecathelinais C. Brief assessment of adult cancer patient's perceived needs: development and validation of the 34-item supportive care needs survey (SCNS-SF34). J Eval Clin Pract. 2009;15:602-6.

25. Osborne RH, Batterham RW, Elsworth GR, Hawkins M, Buchbinder R. The grounded psychometric development and initial validation of the health literacy questionnaire (HLO). BMC Public Health. 2013:13:685.

26. Osborne $\mathrm{RH}$, Elsworth GR, Whitfield $\mathrm{K}$. The health education impact questionnaire (heiQ). An outcomes and evaluation measure for patient education and self-management interventions for people with chronic conditions. Patient Educ Couns. 2007:66:192-201.

27. Given CW, Given B, Stommel M, Collins C, King S, Franklin S. The caregiver reaction assessment (CRA) for caregivers to persons with chronic physical and mental impairments. Res Nurs Health. 1992;15:271-83. 
28. Holmstrom AJ, Wyatt GK, Sikorskii A, Musatics C, Stolz E, Havener N. Dyadic recruitment in complementary therapy studies: experience from a clinical trial of caregiver-delivered reflexology. Appl Nurs Res. 2016;29:136-9.

29. Lambert SD, McElduff P, Girgis A, Levesque JV, Regan TW, Turner J, et al. A pilot, multisite, randomized controlled trial of a self-directed coping skills training intervention for couples facing prostate cancer: accrual, retention, and data collection issues. Supp Care Cancer. 2016;24:711-22.

30. McDonnell KK, Hollen PJ, Heath J, Andrews JO. Recruiting family dyads facing thoracic cancer surgery: challenges and lessons learned from a smoking cessation intervention. Eur J Oncol Nurs. 2016;20:199-206.

31. Sygna $\mathrm{K}$, Johansen $\mathrm{S}$, Ruland CM. Recruitment challenges in clinical research including cancer patients and caregivers. BMC Trials. 2015;16:428-36.

Submit your next manuscript to BioMed Central and we will help you at every step:

- We accept pre-submission inquiries

- Our selector tool helps you to find the most relevant journal

- We provide round the clock customer support

- Convenient online submission

- Thorough peer review

- Inclusion in PubMed and all major indexing services

- Maximum visibility for your research

Submit your manuscript at www.biomedcentral.com/submit 